\title{
Realization of the Radiance Scale Using Transfer Function of the Laser-Based Optical System
}

\author{
M. Kilin $\mathbb{D}^{1},{ }^{1}$ H. Tutunculer, ${ }^{1}$ O. Bazkir, ${ }^{2}$ and S. Meric ${ }^{2}$ \\ ${ }^{1}$ Engineering Physics Department, Gaziantep University, 27310 Sehitkamil, Gaziantep, Turkey \\ ${ }^{2}$ Optic Laboratory, TUBITAK-National Metrology Institute (UME), 41470 Gebze, Kocaeli, Turkey \\ Correspondence should be addressed to M. Kilin; mkilin@gantep.edu.tr
}

Received 2 August 2019; Revised 14 February 2020; Accepted 6 March 2020; Published 1 April 2020

Academic Editor: Nicusor Iftimia

Copyright $\odot 2020$ M. Kilin et al. This is an open access article distributed under the Creative Commons Attribution License, which permits unrestricted use, distribution, and reproduction in any medium, provided the original work is properly cited.

\begin{abstract}
This work aims to determine the radiance responsivity to be used in the calibration of polychromatic radiation sources with low uncertainty. To realize the radiance, Ar-ion, He-Ne, and Nd-YAG lasers as well as an integrating sphere with a $0.15 \mathrm{~m}$ diameter are used to obtain radiation sources having Lambertian distributions. Then, a silicon photodiode-based reflection-type trap detector with calibrated precision aperture, which is traceable to a liquid helium cooled laser-based cryogenic radiometer, is used to measure the photocurrent corresponding to each wavelength and thereby to obtain radiance. The proposed system, which measures the spectral current response of this laser-based radiance, is a double-grating monochromator with a $2 \times 300 \mathrm{~mm}$ focal length and triple gratings in each of its turrets. First, the radiance of the laser beam that emerged from the integrating sphere is calculated, and then the radiance responsivity of the system is obtained by measuring the photocurrent outputted from the exit slit of the monochromator at each laser wavelength. Finally, the spectral radiance values of the polychromatic lamps are obtained using the radiance responsivity of the system. Consequently, the study aims to develop the derivation and better understand traceability of the other radiometric and photometric quantities with low uncertainty from the fundamental radiometric radiance unit. Measurement results obtained in the expanded measurement uncertainty scale are determined using both classical and Monte Carlo methods.
\end{abstract}

\section{Introduction}

In optic radiometry, measurement systems consist of three main components, namely, radiation sources, detectors, and optic and optomechanical materials, which form the basis for the environment in which they are transferred. The measurement traceability chain for the control of the optical performance of these components can be realized in two different ways, depending on whether they are source or detector based.

Source-based measuring chains employ the blackbody radiation principle, are called "black body radiators," and are formed to be traceable to high-precision systems [1, 2]. Detector-based measuring chains have been formed such that they are traceable to low-temperature radiometry that operates on the principle of electrical substitution of the response of the detectors to optical power at the liquid helium temperature $(4.2 \mathrm{~K})$ [3-6].
One of the methods utilized in measurement chains that are developed to realize the radiation scale is to obtain the radiation of the light source compared with that of black body radiator systems. These radiation measurements are performed at specific temperatures by extending with physical modeling of the spectral radiation data [7-9]. Another alternative measurement method that was developed uses a monochromator system and a sensor that is calibrated against a low-temperature radiometer operating at liquid helium temperature $(4.2 \mathrm{~K})[10,11]$. The second one has a lower uncertainty than the first, but it is within the required resolution range for the measurements; the signalto-noise ratio (SNR) of the output of the monochromator causes high uncertainty in the transfer function. In recent years, a method that was developed to solve this problem, and which has been used in metrology laboratories, is the use of a laser radiation source instead of a polychromatic 
radiation source. This approach has helped to obtain the radiation scale and to determine the monochromator transfer function with low uncertainty. By using laser radiation, the abovementioned problems can be greatly reduced as more stable, narrow-band, and high-powered beams can be obtained compared with polychromatic radiation sources [12-16].

In this study, laser-based high-resolution spectroradiometric systems were established. The proposed system calculates the basic radiometric unit radiance with low uncertainty and meets the needs of instrument characterization, which may occur in scientific research activities using other radiometric and photometric quantities. In addition, it aims to establish the infrastructure for potential research needs that may emerge under various topics within the scope of the European Metrology Program for Innovation and Research (EMPIR).

The spectral radiance responsivity scale obtained with low uncertainty for source-based measurement systems [17] can be used for applications such as space and defense applications. The aim of this paper is to develop a spectral radiance responsivity scale by investigating and establishing a suitable radiance measurement system. In addition, the spectral radiance of the unknown radiation source or surface can be defined using a laser-based radiation source, which has a Lambertian distribution. The defined measurement results were calculated separately using both classic and Monte Carlo methods, and they were compared with each other.

In this research, a monochromator that is used for the spectral radiance measurement was first characterized in terms of the wavelength accuracy [18]. Secondly, a reflection-type Si-based trap detector, which is used in the NMI laboratory as a radiometric transfer standard $[19,20]$, was regenerated and comparatively tested based on the homogeneity, polarization dependence, nonlinearity, quantum efficiency, and responsivity using a reflectiontype Si-based trap detector, which was previously characterized against an electrical substitution cryogenic radiometry (ESCR) system in the TÜBİTAK-UME optic laboratory facility $[21,22]$. Thirdly, by using an integrating sphere and laser, the Lambertian-distributed radiance beam was determined. The integrating sphere is a nearly Lambertian source that has the same radiance field in all directions, so it is generally suitable for radiance and irradiance measurements [15]. In the specific laser wavelength region, radiance values of the laser beam created with the integrating sphere were obtained using a $\mathrm{He}-\mathrm{Ne}$ tunable $(543,594,604,612$, and $632.8 \mathrm{~nm})$, Argon-ion (457, 477,488 , and $514 \mathrm{~nm}$ ), and Nd-YAG (1064 nm) lasers. After that, using a monochromator, detector, and polychromatic light, radiance responsivity values were measured. Spectral radiance values obtained by using certain laser wavelengths and trap detectors have been transferred to the system consisting of telescope, monochromator, and detector and transfer function has been produced. These calculations and operations performed at certain laser wavelengths were checked using a reference standard polychromatic radiation source, and the scale was generalized by fitting to the points where laser wavelengths were missing. The known radiance values of both laser and reference standard radiation sources provide an important use for pairwise comparison and generalization while creating the transfer function of the system. Since the use of laser for transfer function is within the facilities of the laboratory in terms of metrology, the reference polychromatic source has provided an important step for both customizing and controlling measurement accuracy. By using the obtained radiance values, the transfer function of the monochromator was obtained, and the radiation values of an unknown source were obtained with standard uncertainty measurements using both the classical and Monte Carlo approaches. The radiance is the basic unit for measurements in the optical region, and the radiance is easily converted to another unit. For this reason, the measurement of the radiance is very important for photometric and radiometric measurements.

The absolute radiance responsivity was realized by transferring the radiation that exists from a tunable laser system with an integrating sphere and regenerated reflection-type trap detector to a spectroradiometric system [23-25]. The spectral radiance of the unknown sources was investigated at high resolution by using uncertainty calculations. Laser-based systems are more suitable for realizing the radiance measurement calibration of any source compared to traditional methods $[16,26]$.

\section{Measurement Setup and Theoretical Background}

In radiometry, optical power measurements are important for both radiation sources and optical detectors. The measurement and testing with low uncertainty of the spectral power values of the radiation source and the current values created in the detectors is an important step for researchers who perform scientific studies using such optical systems. In radiometry, a fundamental optical quantity is radiance and other quantities can be computed from it.

In recent years, the method preferred by many metrology laboratories involves the use of optical detectors and laser sources, which are traceable to low-temperature radiometers. The radiance measurement technique that is realized using laser radiation sources and detectors that are traceable to low-temperature radiometry can meet the requirements that will emerge in parallel with current technological developments in this field [12].

In this study, a laser-based measurement system was established, as shown in Figure 1. Firstly, spectral radiance values were obtained at the laser wavelength by using a silicon-based trap detector, which is characterized with respect to low-temperature radiometry [27]. Then, the same system was transferred to the double monochromator system, and the spectral transfer function of the system was obtained. Thus, the spectral radiance values of the unknown polychromatic radiator source were derived using a transfer function obtained with low uncertainty.

The left part of Figure 1, which has lasers, an integrating sphere, Si-based trap detector, amplifier, and multimeter, was used to obtain the spectral radiance values for each laser 


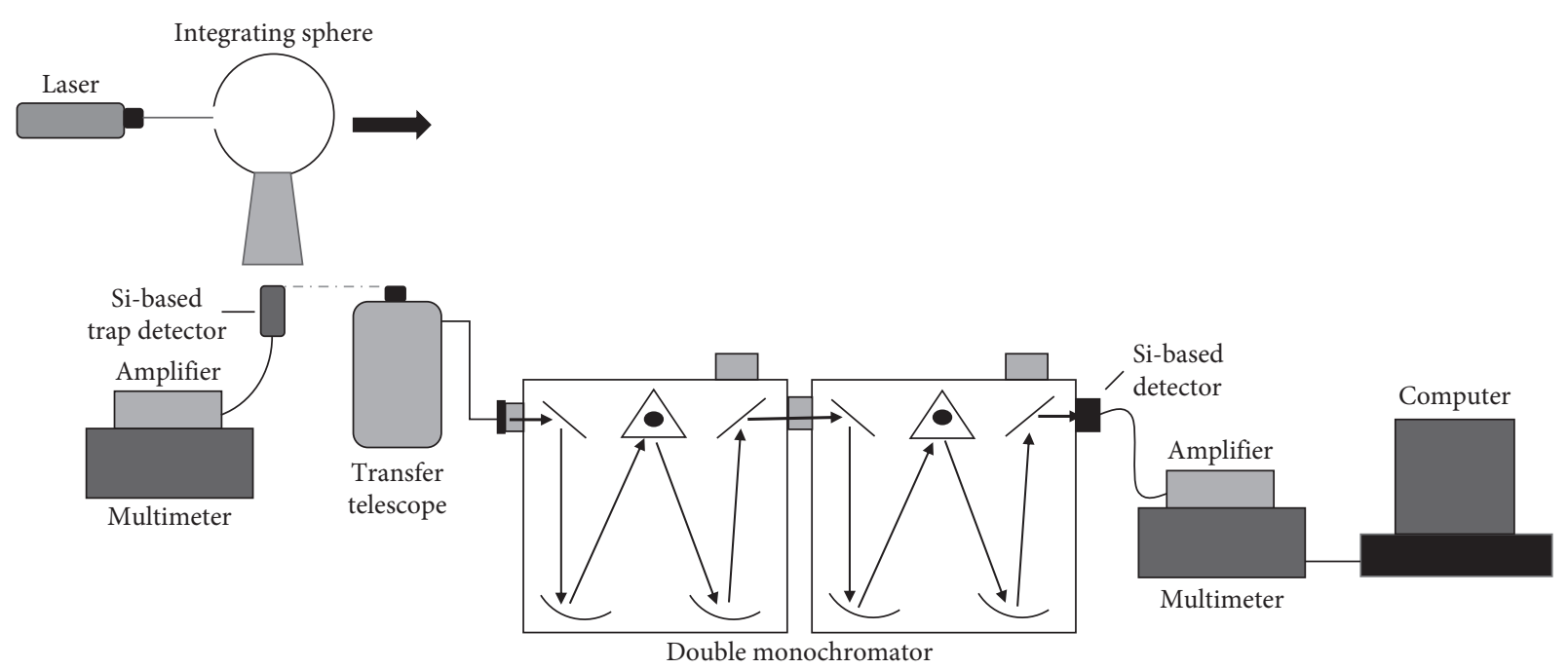

FIGURE 1: Spectral radiance measurement system.

wavelength. Radiance was obtained numerically by analyzing the physical parameters between the source-detector in the measurement apparatus of Figure 1. Theoretically, the following method was used to obtain the radiance formula [28].

The relation between the radiation flux $(\varnothing)$ and the radiance $(L)$ from the radiation source is given as follows:

$$
\mathrm{d}^{2} \varnothing(\lambda)=L(\lambda) \frac{\mathrm{d} A_{1} \cos \theta_{1}}{\left(x_{2}-x_{1}\right)^{2}} \mathrm{~d} A_{2} \cos \theta_{2} .
$$

Radiation flux defined in (1) is a function of the spatial and angular coordinates. Often source of radiometric interest can be considered Lambertian. They radiate a constant radiance in all directions into hemisphere. Because of that reason, some assumptions can be made and integral only can depend on function of the wavelength [28]. Because laser radiation is very similar to monochromatic radiation, when the integral of the delta function is taken over the wavelength of the laser, spectral radiance may lose its integral dependence and it can be obtained by using integrating sphere with narrow-band laser radiation. follows:

The current $I^{s}$ generated in the detector is expressed as

$$
I^{s}=\int R_{s}^{*}(\lambda) \varnothing(\lambda) \mathrm{d} \lambda,
$$

where $R_{s}^{*}(\lambda)$ is the spectral power responsivity of the trap detector, and by considering the required parameters and geometric values in Figure 2, the relation between the spectral radiation and the radiation flux is the same as follows:

$$
\begin{aligned}
I^{s} & =\frac{A_{s} A_{d}(1+\delta)}{\left(r_{s}^{2}+d^{2}+r_{d}^{2}\right)} \int L(\lambda) R_{s}^{*}(\lambda) \mathrm{d} \lambda, \\
L(\lambda) & =\frac{I^{S}}{R_{s}^{*}(\lambda)} \frac{D^{2}}{A_{s} A_{d}(1+\delta)},
\end{aligned}
$$

where $\delta=r_{s}^{2} r_{d}^{2} / D^{4}$ and $D^{2}=r_{s}^{2}+d^{2}+r_{d}^{2}$ in (3) and $A_{s}$ and $A_{d}$ are the active area of the radiation source and

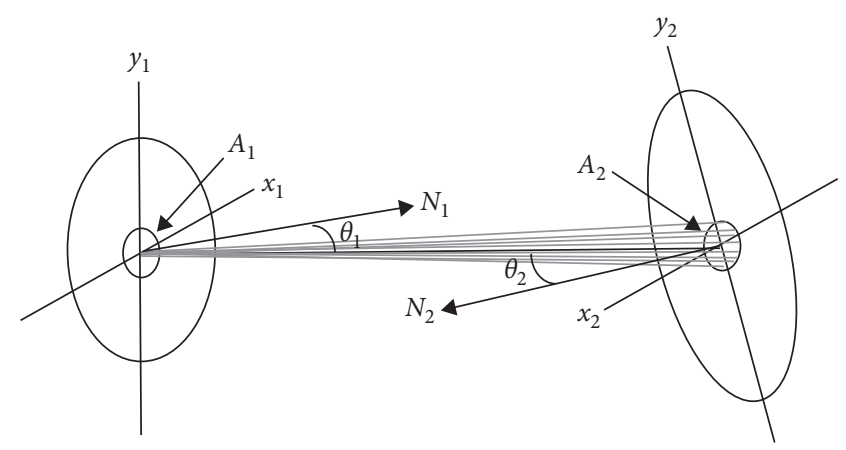

FIGURE 2: Geometric identification between source and detector.

detector, respectively. $d$ represents the distance between the radius of source $r_{s}$ and detector $r_{d}$. In addition, $I^{s}$ is the measured spectral current, $L(\lambda)$ is the spectral radiance, and $R_{s}^{*}(\lambda)$ is the spectral power responsivity of the detector.

2.1. Model Used for Evaluation of the Laser-Based Radiance Measurements. When a measurement is made to determine the radiation power, the relationship between the output signal and the power responsivity of a standard detector is given as in (2). Using geometric and radiometric calculations, (3) was obtained. By using (2) and (3), and some reductions, (4) was then obtained:

$$
C=\frac{A_{s} A_{d}(1+\delta)}{\left(r_{s}^{2}+d^{2}+r_{d}^{2}\right)},
$$

$$
L(\lambda)=\frac{\mathrm{d} \varnothing(\lambda)}{\mathrm{d} C}
$$

The spectral radiance responsivity $R_{s}(\lambda)$ can be changed using spectral power responsivity, $R_{s}^{*}(\lambda)$; with $R_{s}(\lambda)=R_{s}^{*}(\lambda) \Delta C$ and $(\varnothing(\lambda)) / \Delta C=(\mathrm{d} \varnothing(\lambda)) / \mathrm{d} C$, the standard detector output signal can be obtained: 


$$
I^{S}=\int R_{s}(\lambda) L(\lambda) \mathrm{d} \lambda
$$

where $I^{s}(\lambda)$ is given in Ampere, $R_{s}(\lambda)$ in $\mathrm{A} /\left(\mathrm{W} /\left(\mathrm{m}^{2} \cdot \mathrm{sr} \cdot \mathrm{nm}\right)\right)$, and $L(\lambda)$ in $\mathrm{W} /\left(\mathrm{m}^{2} \cdot \mathrm{sr} \cdot \mathrm{nm}\right)$. Because laser radiation is very similar to monochromatic radiation, when the integral of the delta function is taken over the wavelength of the laser, the output signal of the detector is obtained as follows:

$$
I^{s}=\frac{R_{s}\left(\lambda_{L}\right)}{\Delta C} \Delta \varnothing(\lambda)
$$

where $\Delta \varnothing(\lambda) / \Delta C=L_{\text {Laser, } \lambda_{L}}$ is the laser radiance for the spectral line at wavelength $\lambda_{L}$. Note that $I^{s}=I_{\text {Detector }}-I_{\text {Detector, Background }}$.

When the radiance field that is obtained is transferred to the monochromator, the output signal of the monochromator is dependent on the laser wavelength and the adjusted wavelength of the monochromator. The obtained signal becomes the spectral radiance responsivity function of the monochromator $R_{M}\left(\lambda_{M}, \lambda_{L}\right)$. Thus, the output signal of the monochromator $I^{M}$ is described as follows:

$$
I^{M}=\int R_{M}\left(\lambda_{M}, \lambda_{L}\right) L_{\text {Laser }, \lambda_{L}} \mathrm{~d} \lambda .
$$

Substituting for $L_{\text {Laser, } \lambda_{L}}$ in (7), the following equation for the unknown spectral radiance responsivity of monochromator $R_{M}\left(\lambda_{M}, \lambda_{L}\right)$ holds:

$$
R_{M}\left(\lambda_{M}, \lambda_{L}\right)=\frac{I^{M} R_{s}\left(\lambda_{L}\right)}{I^{S}}=\frac{I^{M} R_{s}^{*}\left(\lambda_{L}\right) \Delta C}{I^{S}} .
$$

The radiance field that is obtained was transferred to the monochromator in three different fields of view (1.7 mrad, $5 \mathrm{mrad}$, and $11 \mathrm{mrad})$. A Bentham Tel310 telescope was used during the transmission of the radiance field. Therefore, the spectral radiance transfer function of the monochromator system was defined based on the telescope, fiber-optic cable, double monochromator, and detector parameters.

The monochromator system to be defined as a transfer function is a double-case system, so three slit structures are available, namely, the entrance, middle, and exit slits. In order for the transmitted radiance field to give the correct output signal, each slit range requires a suitable adjustment. Both in theory and practice, slit ranges can be set to the same values. However, in double monochromator systems, the entrance slit should be wider than the exit slit, and the middle slit should be wider than the entrance slit. The differences between the slit widths should not be significant. Each of the three slits has its own special significance. Therefore, as the exit slit is at the side where the image occurs, it needs a maximum SNR in the smallest slit value [17].

The radiance field resulting from a light source is transformed into an output signal depending on the wavelength of the monochromator $\lambda_{M}$, radiation source $\lambda_{L}$, and the spectral radiance responsivity function of the monochromator $R_{M}\left(\lambda_{M}, \lambda_{L}\right)$. Thus, if (7) is rearranged by using the output signal $I^{U}$ of the unknown source from the monochromator and the wavelength adjustment of the monochromator $\lambda_{M}$, so $R_{M}\left(\lambda_{M}, \lambda_{L}\right) \longrightarrow R_{M}\left(\lambda_{M}, \lambda_{M}\right)$, the result can be obtained as follows:

$$
I^{U}=\int R_{M}\left(\lambda_{M}, \lambda_{M}\right) L^{U}(\lambda) \mathrm{d} \lambda,
$$

where $I^{U}$ is the monochromator output signal at the monochromator wavelength setting $\lambda_{M}$ and $L^{U}(\lambda)$ is the spectral radiance of the unknown source. Because $L^{U}(\lambda)$ has a linear dependence as a function of the wavelength, $L^{U}(\lambda)$ can be moved outside the integral.

The spectral radiance responsivity of the monochromator is taken as the integral over the wavelength setting, and when (8) is used, (10) was obtained for the radiance value of the unknown source:

$$
L^{U}(\lambda)=\frac{I^{U} I^{S}}{I^{M} R_{s}^{*}\left(\lambda_{L}\right) \Delta \lambda \Delta C},
$$

where $\Delta \lambda$ is the full width at half maximum (FWHM), which is the spectral band-pass value of the monochromator. The slit spacing of the double monochromator system was adjusted to calculate this band-pass value.

2.2. System Characterization and Correction Factors. The spectral radiance formula defined in (10) should include some uncertainty and correction factors caused by the measurement setup in Figure 1. These are the wavelength accuracy of the monochromator system, trap detector characterization, geometric correction factor owing to the conservation of radiance, the monochromator wavelength shift, and the bandwidth.

The first of these correction parameters is the wavelength uncertainty of the monochromator. For each grating system in the double-case monochromator system, the specified wavelength uncertainty of the scale is determined as in Table 1 [18].

Spectral optical power responses of the regenerated reflection-type silicon-based trap detector were obtained and compared with the trap detector, which was characterized using electrical substitution low-temperature cryogenic radiometry in the National Metrology Institute of Turkey (UME) facility [27].

The spectral power responsivity that is obtained for different optical power values gives us information about the detector's linearity and homogeneity. The measurement uncertainty for the detector in the $k=2$ expanded measurement uncertainty was calculated as $1.5 \%$ in the wavelength range of $300 \mathrm{~nm}$ to $340 \mathrm{~nm}, 1.1 \%$ in the wavelength range of $350 \mathrm{~nm}$ to $850 \mathrm{~nm}$, and $1.4 \%$ in the wavelength range of $860 \mathrm{~nm}$ to $1100 \mathrm{~nm}$.

Another characterization parameter is the geometric correction factor, which is described according to the radiance conservation [28]. The approximate Lambertian radiation pattern that was obtained using the integrating sphere and the He-Ne $632.8 \mathrm{~nm}$ laser was measured by the trap detector at regular intervals from $0.05 \mathrm{~m}$ to $0.39 \mathrm{~m}$. At each point, the radiance values were determined by using measured current values and geometric parameters with (3). The equation $y=a \times x^{b}+c$ was derived when the power series 
TABLE 1: Results showing the measurement uncertainty for double monochromator [18].

\begin{tabular}{lcccc}
\hline Monochromator type & $\begin{array}{c}\text { Grating } \\
(\mathrm{g} / \mathrm{mm})\end{array}$ & Spectral band width, SBW $(\mathrm{nm})$ & Result uncertainty $(\mathrm{nm})$ & Expanded \% 95 $(k=2)$ uncertainty $(\mathrm{nm})$ \\
\hline \multirow{3}{*}{ Double } & 2400 & 0.17 & 0.146 & 0.291 \\
& 1200 & 0.34 & 0.138 & 0.275 \\
& 600 & 0.68 & 0.146 & 0.293 \\
\hline
\end{tabular}

second-order curve fit was made with the graph of the radiance values as a function distance. Using the fit function, a specific two-point range where the changes in radiance values are constant was determined, and the new current values were normalized according to these points. Because of the new current values, the geometrical correction factor was found between $1.2392 \pm 0.0156$ and $1.2484 \pm 0.0158$ according to the law of conservation of radiance with a relative standard uncertainty.

When the radiance field that was obtained by using the integrating sphere was transmitted to the double monochromator system, there is a correction factor owing to the wavelength shift of the monochromator [29]. This wavelength shift was characterized as in (11). The radiance field was transmitted along the telescope (Bentham Tel 310) at three different fields of view $(1.7 \mathrm{mrad}, 5.0 \mathrm{mrad}$, and $11.0 \mathrm{mrad})$ to the monochromator system defined as in Figure 1:

$$
\operatorname{CorFac}_{W S}=\frac{\left(1-\Delta \lambda_{W C} I^{\prime}\right) / I}{\left(1-\Delta \lambda_{W C} I_{T}^{\prime}\right) / I_{T}},
$$

where CorFac $W S$ is the correction factor of the wavelength shift. Here, $I$ and $I_{T}$ are the current values on the output of the double monochromator and trap detector, respectively. In addition, the quotation mark $I^{\prime}$ represents the first derivation of $I$ by wavelength. The derivative operation was performed by applying numerical derivative rules. Interpolation and central difference approximation operations were performed, and the numerical derivative was applied to certain wavelength values. Because of the calculations for each field of view and the wavelength, the correction factors that were determined by the wavelength shift are defined as given in Table 2. In addition, the wavelength correction is $\Delta \lambda_{W C}=\Delta \lambda_{1}+\Delta \lambda_{2}$, where $\Delta \lambda_{1}$ is the result of the offset from the wavelength of the monochromator and has a different value for each wavelength. The value defined as $\Delta \lambda_{2}$ is identical for each wavelength and is caused by system repeatability.

Another correction factor for the monochromator bandwidth is called the CorFac $\mathrm{BW}_{\mathrm{BW}}$. This definition is given in (12), which corrects the triangle slit function of the monochromator [29]. $I^{\prime \prime}$ refers to the $2^{\text {nd }}$ derivation of the current as a function of the wavelength. For different fields of view, it is calculated as in Table 3:

$$
\operatorname{CorFac}_{B W}=\frac{\left(1-\Delta \lambda_{W C}^{2}(1 / 12) I^{\prime \prime}\right) / I}{\left(1-\Delta \lambda_{W C}^{2}(1 / 12) I_{T}^{\prime \prime}\right) / I_{T}} .
$$

For correction factor measurements that are based on the wavelength shift and bandwidth, the reason for which the uncertainties are smaller in large mrad values than the small mrad values besides the radiation intensities of different laser sources is different for each laser wavelength. The increase in the uncertainty of laser sources with low radiation intensity at a low FOV is due to a reduction in the radiation intensity when passing through the integrating sphere and through the monochromator system.

If the spectral radiance distribution formula of an unknown radiation defined as in (10) is redefined, it is given as follows:

$$
L^{U}(\lambda)=\frac{I^{U} I^{S}}{I^{M} R_{s}^{*}\left(\lambda_{L}\right) \Delta \lambda \Delta C} \text { CorFac. }
$$

\section{Spectral Radiance Responsivity of Measurement System}

When (8) and (13) are reconsidered, the spectral radiance responsivity value of the measurement system is reproduced as a function of a known radiation source. The formula defined in (8) is the spectral radiance responsivity value obtained by using laser-based radiation. By separately applying these two equations, spectral radiance responsivity values were obtained for the same system. In (14), the Bentham SRS-12 reference standard radiation source was used, and with its value, the responsivity value of the measurement device consisting of Tel310, fiber-optic cable, and a double monochromator system was generated spectrally. $L^{\wedge}\left(\lambda_{M}\right)$ and $I^{\wedge}$ are the certificate radiance value and the current value of the monochromator output signal, respectively, as defined in (14). The SRS-12 reference standard has radiance certification values at $5 \mathrm{~nm}$ interval wavelength compared to the source laser wavelengths. The radiance values at these points were compared with the expanded uncertainty values with the laser-based system and generalized to the branch length region of $300 \mathrm{~nm}$ and $1100 \mathrm{~nm}$. The use of the SRS-12 reference radiation source alone for the system to define the transfer function is not accurate for the control of traceability from a metrological point of view. Therefore, it is more accurate to use SRS-12 only for generalizing and solidifying measurements:

$$
R_{M}^{\wedge}\left(\lambda_{M}, \lambda_{M}\right)=\frac{I^{\wedge}}{L^{\wedge}\left(\lambda_{M}\right) \Delta \lambda} \text { CorFac. }
$$

The spectral radiance responsivity values that were obtained for all three mrad FOV values were investigated in the same wavelength range for both the laser-based measurement system and the SRS12 reference-source measurement system. This definition describes the transfer function for the 
TABLE 2: Wavelength shift correction factor for all mrad field of view (FOV) values.

\begin{tabular}{|c|c|c|c|c|c|c|}
\hline \multirow{2}{*}{ Wavelength } & \multicolumn{2}{|c|}{$1.7 \mathrm{mrad}$} & \multicolumn{2}{|c|}{$5 \mathrm{mrad}$} & \multicolumn{2}{|c|}{$11 \mathrm{mrad}$} \\
\hline & Value & Sigma & Value & Sigma & Value & Sigma \\
\hline 457 & $9.50 E-01$ & $3.53 E-02$ & $9.84 E-01$ & $3.23 E-03$ & $9.95 E-01$ & $3.20 E-04$ \\
\hline 477 & $9.92 E-01$ & $1.35 E-02$ & $9.98 E-01$ & $8.48 E-04$ & $9.99 E-01$ & $4.05 E-04$ \\
\hline 488 & $9.99 E-01$ & $2.56 E-03$ & $1.01 E+00$ & $4.04 E-04$ & $1.01 E+00$ & $3.47 E-03$ \\
\hline 514 & $9.87 E-01$ & $3.58 E-03$ & $9.92 E-01$ & $7.45 E-04$ & $9.99 E-01$ & $2.30 E-04$ \\
\hline 543 & $9.86 E-01$ & $3.00 E-02$ & $9.82 E-01$ & $1.26 E-03$ & $9.91 E-01$ & $5.89 E-04$ \\
\hline 594 & $9.93 E-01$ & $5.07 E-03$ & $9.96 E-01$ & $7.52 E-04$ & $1.00 E+00$ & $5.68 E-04$ \\
\hline 604 & $9.90 E-01$ & $8.34 E-03$ & $9.98 E-01$ & $1.18 E-03$ & $1.00 E+00$ & $1.11 E-03$ \\
\hline 612 & $9.85 E-01$ & $3.89 E-03$ & $9.83 E-01$ & $7.79 E-04$ & $9.87 E-01$ & $4.57 E-04$ \\
\hline 633 & $1.00 E+00$ & $3.82 E-03$ & $9.97 E-01$ & $6.11 E-04$ & $9.99 E-01$ & $2.51 E-04$ \\
\hline 1064 & $1.00 E+00$ & $3.10 E-03$ & $1.00 E+00$ & $8.97 E-04$ & $9.98 E-01$ & $1.38 E-03$ \\
\hline
\end{tabular}

TABLE 3: Monochromator bandwidth correction factor for all mrad FOV values.

\begin{tabular}{|c|c|c|c|c|c|c|}
\hline \multirow{2}{*}{ Wavelength } & \multicolumn{2}{|c|}{$1.7 \mathrm{mrad}$} & \multicolumn{2}{|c|}{$5 \mathrm{mrad}$} & \multicolumn{2}{|c|}{$11 \mathrm{mrad}$} \\
\hline & Value & Sigma & Value & Sigma & Value & Sigma \\
\hline 457 & $1.00 E+00$ & $9.12 E-03$ & $1.00 E+00$ & $2.78 E-03$ & $1.00 E+00$ & $3.68 E-04$ \\
\hline 477 & $1.01 E+00$ & $4.65 E-03$ & $1.00 E+00$ & $7.48 E-04$ & $1.00 E+00$ & $2.32 E-04$ \\
\hline 488 & $1.00 E+00$ & $5.73 E-04$ & $1.00 E+00$ & $5.91 E-05$ & $1.00 E+00$ & $7.35 E-04$ \\
\hline 514 & $1.00 E+00$ & $2.29 E-03$ & $1.00 E+00$ & $3.77 E-04$ & $1.00 E+00$ & $1.37 E-04$ \\
\hline 543 & $9.99 E-01$ & $6.34 E-03$ & $1.00 E+00$ & $2.38 E-03$ & $1.00 E+00$ & $2.67 E-04$ \\
\hline 594 & $1.01 E+00$ & $2.60 E-03$ & $1.00 E+00$ & $3.99 E-04$ & $1.00 E+00$ & $2.81 E-04$ \\
\hline 604 & $9.88 E-01$ & $1.98 E-03$ & $1.00 E+00$ & $3.19 E-04$ & $1.00 E+00$ & $3.37 E-04$ \\
\hline 612 & $1.01 E+00$ & $1.18 E-03$ & $1.01 E+00$ & $4.59 E-04$ & $1.00 E+00$ & $8.29 E-05$ \\
\hline 633 & $1.00 E+00$ & $1.33 E-03$ & $1.00 E+00$ & $1.55 E-04$ & $1.00 E+00$ & $7.98 E-05$ \\
\hline 1064 & $1.00 E+00$ & $3.08 E-04$ & $1.00 E+00$ & $5.64 E-04$ & $1.00 E+00$ & $4.60 E-04$ \\
\hline
\end{tabular}

measurement system consisting of a Tel-310 telescope, fiberoptic cable, the monochromator, and the Si-based trap detector. By using this transfer function, the spectral radiance of an unknown radiation source can be defined and further radiometric and photometric quantities can be transferred. Figure 3 illustrates the overall measurements for all mrad FOV values for which the measurement and results were defined.

3.1. Radiance of SRS12 Reference Source. (13) is the generalized radiance equation for an unknown radiation source. The equation, which includes both (8) and (14), which describes the transfer function of the system, is a general equation. The spectral radiance values of SRS12 with sigma-1 uncertainty and the radiance values obtained by laser-based transfer function were compared to the same range, as seen in Figure 4.

\section{Uncertainty Budget of Spectral Radiance for Unknown Source or Surface}

In order to define parameters such as the spectral radiance, irradiance, and power of an unknown radiation source, the measurement uncertainty needs to be calculated. One of the most common tools used to do this is the Guide to the Expression of Uncertainty in Measurement (GUM), which was published in the last available version in 2008, and is used by many NMI laboratories [30]. These uncertainties may occur during the measurement or by the devices used; in addition, the experience of the person carrying out the measurement may affect this situation.

The Monte Carlo method is a relatively new method and is a useful and practical alternative to the GUM method. Here, the probability density function (PDF) is defined for each measured output quantity and defines the system and includes pseudorandom numbers. This method is described as GUM Supplement-1 by ISO and CIE-2006. The probability function that is produced using mean and sigma values for each measurement output used in the calculations can be defined as Gaussian, rectangular, and any other distribution type. This calculation, which is obtained by generating random numbers, is a practical method because it eliminates the difficulty associated with the partial derivation when defining nonlinear systems. In addition, when performing calculations using the GUM method, it is necessary to include all parameters in the calculation of the model function, so the Monte Carlo uncertainty calculation method offers both a practical and a more precise approach than the classical method.

4.1. Definition of Spectral Radiance Value for GUM Method. In this study, the uncertainties of the measurements, certificate values, and other parameters in the calibration according to the GUM [30] method were evaluated as TYPE$A$ and TYPE-B. The resultant measurement and accompanying systematic or random uncertainty values were calculated and placed separately within the uncertainty budget. Finally, the uncertainty budget was completed by calculating 


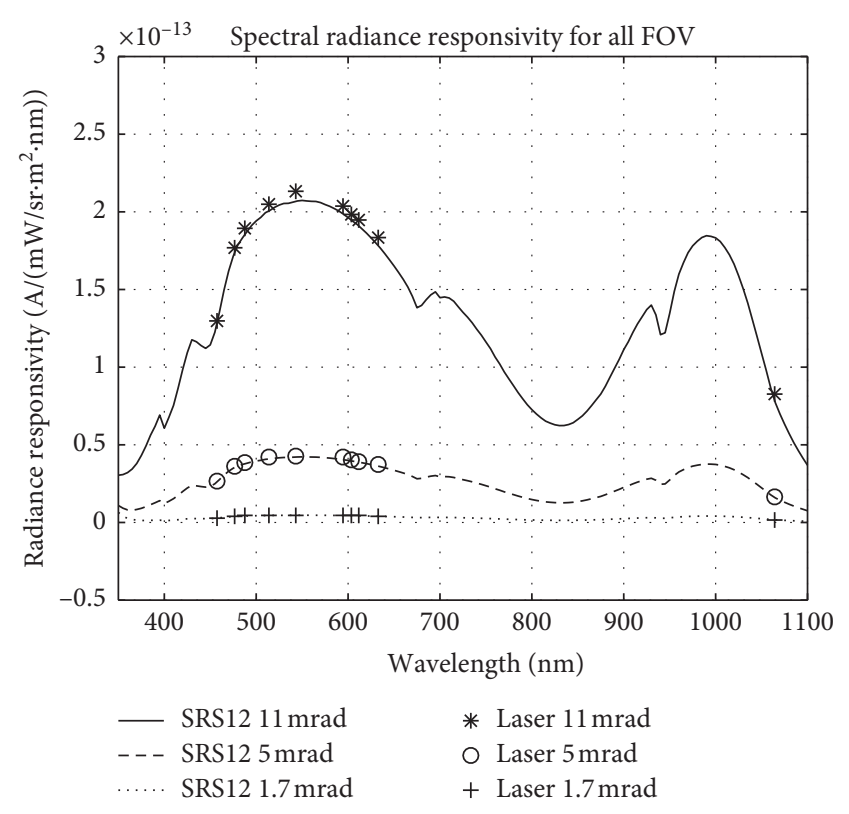

FIGURE 3: Spectral radiance responsivity for both laser-based and reference standard measurement system for all mrad FOV values.

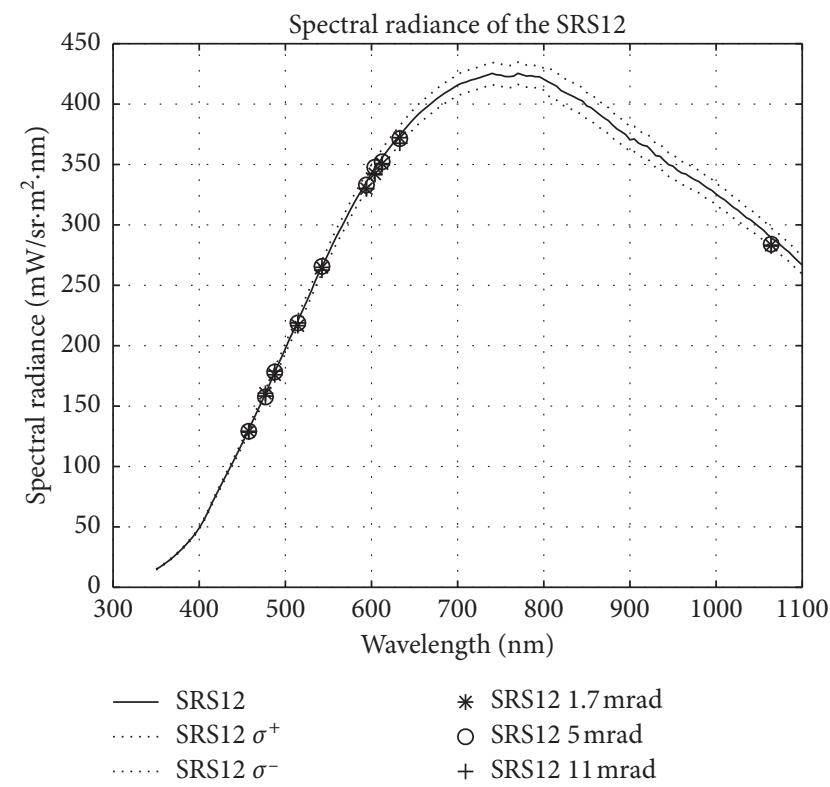

FIGURE 4: Spectral radiance of the SRS12 reference radiation source for all mrad FOV values.

the sensitivity coefficient value. Uncertainty values for each laser wavelength and three mrad FOVs used in the measurement were calculated as a function of the wavelength. Only for the $632.8 \mathrm{~nm} \mathrm{He}-\mathrm{Ne}$ laser wavelength was the uncertainty budget defined in detail, as shown in Table 4, and the spectral radiance values were determined in the $95 \%$ confidence level of the $k=2$ expanded uncertainty.

4.2. Definition of Spectral Radiance Value for GUM Supplement-1 Method. When the function that defines the measurement system is linear, the GUM method offers an easy solution, but when the function that defines the system is not linear, the Monte Carlo [31] method is more practical because the partial derivation of the output quantities according to each input quantity makes the calculations difficult. In addition, all of the uncertainty components in the GUM method need to be defined within the uncertainty budget, and these are more complex than the Monte Carlo method.

Each measurement quantity $y$ and the accompanying $u$ (y) sigma values were defined by a PDF with random numbers according to the type of change, and the sigma value of the Gaussian function formed after its replacement in the function describing the system gives us the associated measurement uncertainty value.

For each mrad FOV, a Monte Carlo operation was performed and the uncertainties corresponding to spectral radiance values at the laser wavelength were calculated. A histogram distribution was also defined for the radiance value obtained for $1064 \mathrm{~nm}$, as shown in Figure 5, and a Gaussian fit function was applied to it.

\section{Results and Discussion}

The spectral radiation values of an unknown light source were determined by defining the spectral transfer function of the measurement system, and the expanded uncertainty value that is associated with the radiance value was defined using both the classical approach and the Monte Carlo approach.

In this study, a laser-based spectral radiance system was established, and this system was transferred to a system that has a telescope, a double monochromator system, and a silicon detector; thus, the spectral radiance transfer function of the system was obtained. By using this transfer function, the spectral radiance value of any radiation source having homogeneous radiation can be obtained.

During the radiance measurements, three different types of gas laser radiation were sent to the integrating sphere, which has a $0.15 \mathrm{~m}$ surrounding diameter, a $0.02 \mathrm{~m}$ exit port diameter, and is covered inside with $\mathrm{BaSO}_{4}$ (barium sulfate has the ability to reflect 99\%). The radiations at the exit port of the integrating spheres have approximately the Lambertian distribution. This radiation having a Lambertian distribution was sent over a reflection-type silicon-based trap detector with an aperture diameter of $0.08 \mathrm{~m}$ from a certain geometric distance to obtain radiance values at each laser wavelength point.

Because of all measurements, a comparative uncertainty calculation for both cases by using both GUM method and Monte Carlo method is given in Table 5. Although there are calculations for each mrad FOV, Table 5 only gives results for $11 \mathrm{mrad}$ values.

These radiance values were transferred to the double monochromator system as a laser-based reference. This was done using a telescope and fiber-optic cable with three different FOV values, namely, 1.7, 5.0, and $11.0 \mathrm{mrad}$. Figure 3 shows that the spectral radiance transfer function was obtained for different FOV values. The results obtained with this measurement have some differences in the FOVs at 
TABLE 4: $k=2$ expanded standard uncertainty budget for $632.8 \mathrm{~nm}$ in the $11 \mathrm{mrad}$ FOV.

\begin{tabular}{|c|c|c|c|c|c|c|c|c|c|}
\hline $\begin{array}{l}\text { Uncertainty } \\
\text { component } \\
\left(X_{i}\right)\end{array}$ & Sym & $\begin{array}{l}\text { Measured } \\
\text { values }\left(x_{i}\right)\end{array}$ & Unit & $\begin{array}{l}\text { Standard } \\
\text { measurement } \\
\text { uncertainty } \\
\quad\left(u\left(x_{i}\right)\right)\end{array}$ & Unit & $\begin{array}{l}\text { Sensitivity } \\
\text { coefficient } \\
\qquad\left(C_{i}\right)\end{array}$ & Unit & $\begin{array}{l}\text { Uncertainty } \\
\text { contribution } \\
\qquad\left(u_{i}(y)\right)\end{array}$ & $\begin{array}{c}\text { Variance } \\
\left(u_{i}^{2}(y)\right)\end{array}$ \\
\hline $\begin{array}{l}\text { Trap detector } \\
\text { responsivity }\end{array}$ & $R^{*}$ & $3.3170 e-01$ & $\mathrm{~A} / \mathrm{W}$ & $3.6487 e-03$ & $\mathrm{~A} / \mathrm{W}$ & $-1.0316 e+00$ & $\mathrm{~W}^{2} / \mathrm{A} \cdot \mathrm{m}^{2} \cdot \mathrm{sr} \cdot \mathrm{nm}$ & $-3.7640 e-03$ & $1.4167 e-05$ \\
\hline $\begin{array}{l}\text { Geometric } \\
\text { constant }\end{array}$ & $\Delta C$ & $6.1421 e-07$ & $\mathrm{~m}^{2} \cdot \mathrm{sr}$ & $1.2536 e-08$ & $\mathrm{~m}^{2} \cdot \mathrm{sr}$ & $-5.5711 e+05$ & $\mathrm{~W} /\left(\mathrm{m}^{2} \cdot \mathrm{sr}^{2} \cdot \mathrm{nm}\right.$ & $-6.9841 e-03$ & $4.8777 e-05$ \\
\hline $\begin{array}{l}\text { Detector } \\
\text { output signal }\end{array}$ & $I^{s}$ & $2.3811 e-07$ & A & $7.8377 e-11$ & $\mathrm{~A}$ & $1.4371 e+06$ & $\mathrm{~W} / \mathrm{A} \cdot \mathrm{m}^{2} \cdot \mathrm{sr} \cdot \mathrm{nm}$ & $1.1263 e-04$ & $1.2687 e-08$ \\
\hline $\begin{array}{l}\text { Spectrometer } \\
\text { output signal }\end{array}$ & $I^{m}$ & $2.8013 e-10$ & A & $1.9801 e-13$ & A & $-1.2215 e+09$ & $\mathrm{~W} / \mathrm{A} \cdot \mathrm{m}^{2} \cdot \mathrm{sr} \cdot \mathrm{nm}$ & $-2.4187 e-04$ & $5.8500 e-08$ \\
\hline $\begin{array}{l}\text { Unknown } \\
\text { output signal }\end{array}$ & $I^{U}$ & $1.7669 e-10$ & A & $6.4885 e-13$ & A & $1.9366 e+09$ & $\mathrm{~W} / \mathrm{A} \cdot \mathrm{m}^{2} \cdot \mathrm{sr} \cdot \mathrm{nm}$ & & $1.5790 e-06$ \\
\hline $\begin{array}{l}\text { Wavelength } \\
\text { band pass }\end{array}$ & $\Delta \lambda$ & $2.6500 e+00$ & $\mathrm{~nm}$ & $1.0000 e-02$ & $\mathrm{~nm}$ & $-1.2912 e-01$ & $\mathrm{~W} / \mathrm{m}^{2} \cdot \mathrm{sr} \cdot \mathrm{nm}^{2}$ & $-1.2912 e-03$ & $1.6673 e-06$ \\
\hline $\begin{array}{l}\text { Correction } \\
\text { factors }\end{array}$ & $\delta R$ & $1.2301 e+00$ & - & $3.7718 e-04$ & - & $1.0000 e+00$ & - & $3.7718 e-04$ & $1.4227 e-07$ \\
\hline Repeatability & $\delta R$ & $3.6793 e-01$ & $\begin{array}{c}\mathrm{W} / \\
\mathrm{m}^{2} \cdot \mathrm{sr} \cdot \mathrm{nm}\end{array}$ & $5.5423 e-04$ & $\begin{array}{c}\text { W/ } \\
\mathrm{m}^{2} \cdot \mathrm{sr} \cdot \mathrm{nm}\end{array}$ & $1.0000 e+00$ & - & $5.5423 e-04$ & $3.0717 e-07$ \\
\hline \multicolumn{9}{|c|}{$\begin{array}{l}\text { Sum }\left(\mathrm{W} / \mathrm{m}^{2} \cdot \mathrm{sr} \cdot \mathrm{nm}\right) \\
\text { Total measurement uncertainty }\left(\mathrm{W} / \mathrm{m}^{2} \cdot \mathrm{sr} \cdot \mathrm{nm}\right) u(L(\lambda)) \\
\text { Expanded measurement uncertainty }\left(\mathrm{W} / \mathrm{m}^{2} \cdot \mathrm{sr} \cdot \mathrm{nm}\right) U(L(\lambda))\end{array}$} & $\begin{array}{l}6.6404 e-05 \\
0.0081 \\
0.0163\end{array}$ \\
\hline
\end{tabular}

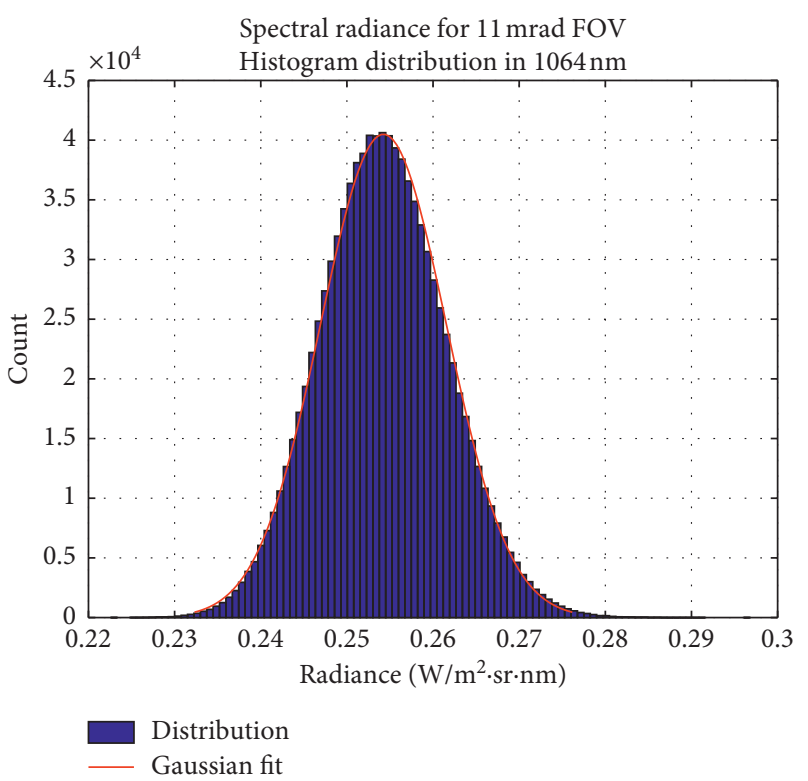

Figure 5: Plot showing $1064 \mathrm{~nm}$ spectral radiance histogram distribution and associated Gaussian fit function. The mean value of the function is the spectral radiance value $0.25424\left(\mathrm{Wm}^{-2} \cdot \mathrm{sr}^{-1} \cdot \mathrm{nm}^{-1}\right)$, and one sigma value of it is $0.007315\left(\mathrm{Wm}^{-2} \cdot \mathrm{sr}^{-1} \cdot \mathrm{nm}^{-1}\right)$.

the same wavelength, which is due to a decrease in laser power values and a decrease in the FOV values.

In order to determine the radiance value of a radiation source, a spectral transfer function that was obtained was used for different FOVs. Figure 4 shows that spectral radiance values were obtained using both the transfer function of the system and certificate values of the SRS12 spectral reference radiance source. The results show that both the certificate and the measurement results have very close values. This measurement setup can be used not only for the radiation source but also for the surface where the radiation reflected.

Because lasers have low power at some wavelengths, the power transmitted to the system at small FOVs will be less, so the uncertainty is high in these regions, as given in Figure 6. However, it is seen that the distribution of uncertainty of the other general regions is between $4.5 \%$ and $6 \%$. 
TABLE 5: $k=2$ expanded standard uncertainty for $11 \mathrm{mrad}$ FOV in terms of both GUM and GUM Supplement-1.

\begin{tabular}{lccccc}
\hline $\begin{array}{l}\text { Wavelength } \\
(\mathrm{nm})\end{array}$ & $\begin{array}{c}\text { Calculated radiance } \\
\left(\mathrm{W} / \mathrm{m}^{2} \cdot \mathrm{sr} \cdot \mathrm{nm}\right)\end{array}$ & $\begin{array}{c}k=2 \text { expanded measurement } \\
\text { uncertainty }\left(\mathrm{W} / \mathrm{m}^{2} \cdot \mathrm{sr} \cdot \mathrm{nm}\right)\end{array}$ & $\begin{array}{c}\text { Calculated } \\
\text { radiance } \\
\left(\mathrm{W} / \mathrm{m}^{2} \cdot \mathrm{sr} \cdot \mathrm{nm}\right)\end{array}$ & $\begin{array}{c}k=2 \text { expanded measurement } \\
\text { uncertainty }\left(\mathrm{W} / \mathrm{m}^{2} \cdot \mathrm{sr} \cdot \mathrm{nm}\right)\end{array}$ & $\begin{array}{c}\text { Ratio of the two } \\
\text { calculations }\end{array}$ \\
\hline 457 & 0.11952 & 0.0058 & 0.11953 & 0.0057 & 1.0229 \\
477 & 0.14892 & 0.0072 & 0.14887 & 0.0071 & 1.0198 \\
488 & 0.16825 & 0.0119 & 0.16819 & 0.0108 & 1.0998 \\
514 & 0.19524 & 0.0094 & 0.19483 & 0.0093 & 1.0086 \\
543 & 0.24632 & 0.0122 & 0.24708 & 0.0121 & 1.0093 \\
594 & 0.32095 & 0.0153 & 0.32041 & 0.0162 & 1.0204 \\
604 & 0.33359 & 0.0165 & 0.33469 & 0.0155 & 0.9980 \\
612 & 0.32635 & 0.0155 & 0.32543 & 0.34237 & 0.0146 \\
1064 & 0.34347 & 0.0163 & 0.25424 & 0.9993 \\
\hline
\end{tabular}

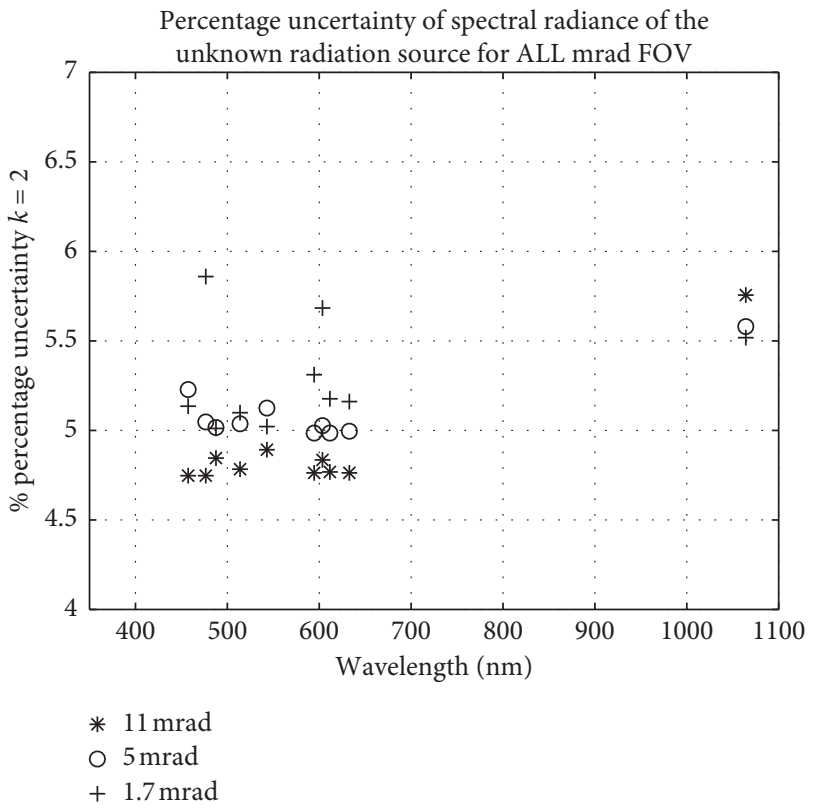

Figure 6: Percentage uncertainty distribution of spectral radiance of the unknown radiation source for all mrad FOV values.

This study was investigated as an internal project in the TUBITAK-UME optical laboratory. In our country's radiometry and photometry fields, it aims to eliminate the dependence on foreign sources by creating a traceability chain. In this way, the correct radiometric and photometric measurements of all radiating materials can be calculated by measuring the conversion to all units with low uncertainty.

\section{Conclusion}

The spectral radiance traceability chain was created, and the radiance value of any radiation source or the surface of an unknown radiance value can be described with ease. In this study, to find a spectral transfer function, uncertainty values were defined by a $95 \%$ confidence level by both the classical method and Monte Carlo method.

This study is a guide towards the derivation of radiometric and photometric measurement units, which are the basis for all optical power measurement systems equipment in laboratories serving Ar-Ge, military, metrology, or industry. In addition, the Monte Carlo uncertainty, which is often preferred by many national metrology laboratories in recent days, is a guide for calculations.

\section{Data Availability}

In this study, the Scientific and Technological Research Council of Turkey (TUBITAK), located within the National Metrology Institute (NMI), is prepared using optical laboratory infrastructure. The study is evaluated within the framework of an internal corporate project. The data used to support the findings of this study are available from the corresponding author upon request.

\section{Conflicts of Interest}

The authors declare that they have no conflicts of interest.

\section{Acknowledgments}

This study was supported by the TUBITAK-National Metrology Institute (UME) Optic Laboratory. The authors thank all researchers of the optic laboratory.

\section{References}

[1] E. R. Woolliams, Development and evaluation of a high temperature blackbody source for the realisation of NPL'S primary spectral irradiance scale, Ph.D. thesis, University of Manchester, Manchester, UK, 2003.

[2] F. Grum and R. Becherer, Optical Radiation Measurements, Vol. 1, Academic Press, Cambridge, MA, USA, 1979.

[3] T. J. Quinn and J. E. Martin, "Blackbody source in the -50 to $+200^{\circ} \mathrm{C}$ range for the calibration of radiometers and radiation thermometers,” Applied Optics, vol. 30, no. 31, p. 4486, 1991.

[4] J. E. Martin, N. P. Fox, and P. J. Key, "A cryogenic radiometer for absolute radiometric measurements," Metrologia, vol. 21, no. 3, pp. 147-155, 1985.

[5] C. C. Hoyt and P. V. Foukal, "Cryogenic radiometers and their application to metrology," Metrologia, vol. 28, no. 3, pp. 163-167, 1991.

[6] O. Bazkir, S. Ugur, F. Samedov, and A. Esendemir, "High-accuracy optical power measurements by using electrical-substitution 
cryogenic radiometer," Optical Engineering, vol. 44, no. 1, pp. 64011-64016, 2005.

[7] H. W. James, D. S. Robert, T. H. Albert, and NBS, "Spectral radiance calibrations," pp. 250-251, National Bureau of Standards, Gaithersburg, MD, USA, 1987, Tech. Rep. 250-1.

[8] P. Sperfeld, J. Metzdorf, S. G. Yousef, K. D. Stock, and W. Möller, "Improvement and extension of the black-bodybased spectral irradiance scale," Metrologia, vol. 35, no. 4, pp. 267-271, 1998.

[9] P. Sperfeld, S. Pape, B. Khlevnoy, and A. Burdakin, "Performance limitations of carbon-cavity blackbodies due to absorption bands at the highest temperatures," Metrologia, vol. 46, no. 4, pp. S170-S173, 2009.

[10] E. W. M. van der Ham, H. C. D. Bos, and C. A. Schrama, "Primary realization of a spectral irradiance scale employing monochromator-based cryogenic radiometry between $200 \mathrm{~nm}$ and $20 \mu \mathrm{m}$," Metrologia, vol. 40, pp. 177-180, 2003.

[11] T. Keawprasert, K. Anhalt, R. D. Taubert, A. Abd-Elmageed, A. Sperling, and J. Hartmann, "Absolute calibration of spectral responsivity for a radiation thermometer," in Proceedings of the 10th International Conference on New Developments and Applications in Optimal Radiometry, Daejeon, South Korea, October 2008.

[12] A. Abd-Elmageed, S. Winter, S. Nevas, P. Sperfeld, S. Kück, and A. Sperling, "Detector based traceability chain for spectral irradiance using tunable lasers, independent from blackbody radiators," in Proceedings of the BulLight 2010, Varna, Bulgaria, June 2010.

[13] T. Keawprasert, K. Anhalt, R. D. Taubert, A. Abd-Elmageed, A. Sperling, and J. Hartmann, "Monochromator based absolute calibration of radiation thermometers," in Proceedings of the TEMPMEKO and ISHM 2010, Portorož, Slovenia, May 2010.

[14] V. E. Anderson, N. P. Fox, and D. H. Nettleton, "Highly stable, monochromatic and tunable optical radiation source and its application to high accuracy spectrophotometry," Applied Optics, vol. 31, no. 4, p. 536, 1992.

[15] S. Brown, G. Eppeldauer, and K. R. Lykke, "Facility for spectral irradiance and radiance responsivity calibrations using uniform sources," Applied Optics, vol. 45, no. 32, p. $8218,2006$.

[16] G. P. Eppeldauer, S. W. Brown, T. C. Larason, M. Rácz, and K. R. Lykke, "Realization of a spectral radiance responsivity scale with a laser-based source and Si radiance meters," Metrologia, vol. 37, no. 5, pp. 531-534, 2000.

[17] A. Abd-Elmageed, Detector-based traceability chain for spectral irradiance using tunable laser-based facility at PTB, Ph.D. thesis, Braunschweig, Germany, 2011.

[18] M. Kilin, H. Tutunculer, and S. Meric, "Effective measurement of the wavelength Accuracy calibration by using Monte Carlo uncertainty calculation," in Proceedings of the AIP Conference Proceedings, vol. 2178, Article ID 030008, Kannur, India, June 2019.

[19] N. Mart, "Development of detectors and calibration methods for spectral irradiance and radiometric temperature measurement," Report 25/2005, Metrology Research Institute, Canada, 2005.

[20] R. Köhler, R. Goebel, and R. Pello, "Results of an international comparison of spectral responsivity of silicon photodetectors," Metrologia, vol. 32, pp. 463-468, 1995.

[21] A. R. Schaefer and K. L. Eckerle, "Spectrophotometric tests using a dye-laser-based radiometric characterization facility," Applied Optics, vol. 23, no. 2, pp. 250-256, 1984.
[22] J. Geist, E. F. Zalewski, and A. R. Schaefer, "Spectral response self-calibration and interpolation of silicon photodiodes," Applied Optics, vol. 19, no. 22, pp. 3795-3799, 1980.

[23] N. P. Fox, "Trap detectors and their properties," Metrologia, vol. 28, no. 3, pp. 197-202, 1991.

[24] R. Friedrich and J. Fischer, "New spectral radiance scale from $220 \mathrm{~nm}$ to $2500 \mathrm{~nm}$," Metrologia, vol. 37, no. 5, pp. 539-542, 2000.

[25] B. Ozcan and S. Ferhad, "Characterization of silicon photodiode-based trap detectors and establishment of spectral responsivity scale," Optics and Lasers in Engineering, vol. 43, no. 2, pp. 131-141, 2005.

[26] S. W. Brown, G. P. Eppeldauer, and K. R. Lykke, "NIST facility for spectral irradiance and radiance responsivity calibrations with uniform sources," Metrologia, vol. 37, no. 5, pp. 579-582, 2000.

[27] B. Ozcan and S. Ferhad, "Electrical substitution cryogenic radiometer based spectral responsivity scale between 250$2500 \mathrm{~nm}$ wavelengths," Optics and Lasers in Engineering, vol. 34, pp. 427-438, 2004.

[28] A. C. Parr, R. U. Dotta, and J. L. Gardner, "Optical Radiometry," Experimental Methods in the Physics Science, Elsevier, vol. 41, Amsterdam, Netherlands, 2005.

[29] S. Winter and A. Sperling, "Uncertainty analysis of a photometer calibration at the DSR setup of the PTB," in Proceedings of the 2nd Expert Symposium on Measurement Uncertainty, CIE x029: 2006, pp. 139-142, Braunschweig, Germany, June 2006.

[30] BIPM Joint Committee for Guides in Metrology, Evaluation of Measurement Data-Guide to Expression of the Uncertainty in Measurement (GUM 1995 Minor Corrections), Vol. 100, BIPM Joint Committee for Guides in Metrology, Sèvres, France, 2008.

[31] BIPM Joint Committee for Guides in Metrology, "Evaluation of Measurement data-Supplement 1 to the Guide to Expression of the Uncertainty in Measurement"-Propagation of Distribution Using a Monte Carlo Method, BIPM Joint Committee for Guides in Metrology, Sèvres, France, 2006. 